\title{
PATIENT HANDOFF
}

Patient handoff (or sign-out) refers to the specific interaction, communication, and planning required to achieve seamless transitions of care from one clinician to another. Effective and timely sign-outs are essential to maintain high quality medical care, reduce medical errors and redundancy, and prevent loss of information. Hospitalists are involved in the transfer of patient care on a daily basis and can lead institutional initiatives that promote optimal transfer of information between health care providers.

\section{KNOWLEDGE}

Hospitalists should be able to:

- Describe key elements involved in signing out a patient.

- Explain important information that should be communicated during patient sign-out, which may include administrative details, updated clinical status, tasks to be completed and relative priority, severity of illness assessment, code status, and contingency planning.

- Explain the components and strategies that are critical for successful communication during sign-outs.

- Explain how the components, strategies and specific information provided at sign-out might vary depending on complexity of the patient, familiarity of provider with the patient and the care environment, and timing of signout.

- Explain the strengths and limitations of various sign-out communication strategies and procedures.

\section{SKILLS}

Hospitalists should be able to:

- Communicate effectively and efficiently during patient sign-out.

- Demonstrate the use of read back when communicating tasks.

- Utilize the most efficient and effective verbal and written communication modalities.

- Construct patient summaries for oral and written delivery, incorporating the unique characteristics of the patient, provider and timing of the sign-out.

- Evaluate all medications for indication, dosing, and planned duration at the time of sign-out.

- Document updated clinical status, recent and pending test and study results, a complete problem list, and plans for continued care.

- Explain the importance of using "if-then" statements for critical tasks to be completed.

- Anticipate what may go wrong with a patient after a transition in care and communicate this clearly to the receiving clinician.

- Synthesize medical information received from Hospitalists signing out patients into care plans

\section{ATTITUDES}

Hospitalists should be able to:

- Inform patients and families in advance of sign-out.

- Recognize the impact of effective and ineffective sign-outs on patient safety.

- Appreciate the value of real time interactive dialogue between hospitalists during sign-out.

- Review received sign-out summaries and communications information carefully and request clarification when needed.

- Engage stakeholders in hospital initiatives to continuously assess the quality of sign-outs.

- Lead, coordinate or participate in initiatives to develop and implement new protocols to improve and optimize sign-outs.

- Lead, coordinate or participate in evaluation of new strategies or information systems designed to improve signouts.

- Promote availability after sign-outs should questions arise. 